\title{
Euler-Lagrange equations for holomorphic structures on twistorial generalized Kähler manifolds
}

\author{
Zeki Kasap \\ Elementary Mathematics Education Department, Pamukkale University, Denizli, Turkey \\ Received: 23 November 2015, Revised: 7 December 2015, Accepted: 22 December 2015 \\ Published online: 10 February 2016.
}

\begin{abstract}
The paper aims to introduce some partial differential equations on Twistorial generalized Kähler manifolds, with an emphasis on Euler-Lagrange equations. Twistor spaces are certain complex 3-manifolds which are associated with special conformal Riemannian geometries on 4-manifolds. Also, classical mechanic is one of the major subfields for mechanics system. A mechanical system has a state determined by a collection of real numbers, or more generally by a set of points in an appropriate state space. Euler-Lagrange equations are an efficient use of classical mechanics to solve problems using mathematical modeling. In this study, showing motion modeling partial differential equations have been obtained for movement of objects in space and solutions of these equations have been generated by using the Maple software. Additionally, of the implicit solution of the equations to be drawn the graph.
\end{abstract}

Keywords: Twistor, Kählerian manifold, mechanical system, dynamic equation, almost complex, lagrangian formalism.

\section{Introduction}

Dynamic systems are a recent theoretical and applied approach to the study of development. In its contemporary formulation, the theory grows directly from advances in understanding complex and nonlinear systems in mathematical physics and differential geometry. A dynamical system is a smooth action of the real or the integers on another object that it named usually a manifold. At any given time of a dynamical system has a state given by a set of a vector that can be represented by a point in an appropriate state space. Also, dynamical systems theory attempts to encompass all the possible factors that may be in operation at any given developmental moment; it considers development from many levels and time scales. Classical mechanics has provided effective solution methods using Euler-Lagrange equations for dynamic systems. Euler-Lagrange equation is one of these methods and it is a model that shows the movement over time of dynamic systems of quantum mechanics. Twisted geometries are discrete geometries that plays a role in loop quantum gravity and spin foam models, where they appear in the semiclassical limit of spin networks and a spin network is a type of diagram which can be used to represent states and interactions between particles and fields in quantum mechanics. Twistor theory was created by Penrose [1]. Penrose created the twistor theory to solve problems in mathematical physics [2]. Freidela and Speziale showed that the phase space of loop quantum gravity on a fixed graph can be parametrized in terms of twisted geometries, quantities describing the intrinsic and extrinsic discrete geometry [3]. Speziale introduced that loop quantum gravity and twisted geometries [4]. Santa-Cruz presented the hyperkähler geometry of complex adjoint orbits from the point of view of twistor theory [5]. Albuquerque used twistor theory to describe virtually new constructions of Hermitian and quaternionic Kähler structures on tangent bundles [6]. Davidov and Mushkarov introduced that the twistor construction is applied for obtaining examples of generalized complex structures [7]. Loubeau and Pantilie examined that Weyl spaces provide a natural context for harmonic morphism [8]. Pantilie shown that a 
natural class of twistorial maps gives a pattern for apparently different geometric maps, such as, (1,1)-geodesic immersions from $(1,2)$-symplectic almost Hermitian manifolds and pseudo horizontally conformal submersions with totally geodesic fibres for which the associated almost CR-structure is integrable [9]. Ianus et al introduced a natural notion of quaternionic map between almost quaternionic manifolds [10]. Dunajski examined an elementary and self-contained review of twistor theory as a geometric tool for solving non-linear differential equations.[11]. Marchiafava obtained that an alternative proof of a characterization of twistorial maps between quaternionic projective spaces [12]. Kasap found Weyl-Euler-Lagrange equations of motion on flat manifold [13]. Tekkoyun revealed Euler-Lagrange and Hamiltonian equations on $\mathbb{R}_{n}^{2 n}$ which is a model of para-Kählerian manifolds of constant J-sectional curvature [14]. Cecotti et al defined twistorial topological strings by considering $\mathrm{tt}^{*}$ geometry of the $4 \mathrm{~d} \mathrm{~N}=2$ supersymmetry theories on the Nekrasov-Shatashvili $\frac{1}{2} \Omega$ background [15].

\section{Preliminaries}

Definition 1. A Hermitian form on a vector space $V$ over the complex field $\mathbb{C}$ is a function $f: V \times V \rightarrow \mathbb{C}$ such that for all $u, v, w$ in $V$ and all $a, b$ in $\mathbb{R}, 1 . f(a u+b v, w)=a f(u, w)+b f(v, w) .2 . f(u, v)=\bar{f}(v, u)$. Here, the bar indicates the complex conjugate. It follows that $f(u, a v+b w)=\bar{a} f(u, v)+\bar{b} f(u, w)$, which can be expressed by saying that $f$ is antilinear on the second coordinate.

Definition 2. Let $\vec{X}=\left(x_{i}\right), \mathbf{Y}=\left(y_{i}\right) \in \mathbb{R}^{3}$ be any two vectors. As follows

$$
<,>: \mathbb{R}^{3} \times \mathbb{R}^{3} \rightarrow \mathbb{R}^{1}, \quad<\vec{X}, \mathbf{Y}>_{L}=-x_{1} y_{1}+x_{2} y_{2}+x_{3} y_{3}
$$

in the form of a function. This function are bilinear and symmetric. This the inner product function $\left\langle X, Y>_{L}\right.$ along with $\mathbb{R}^{3}$ is called Minkowski space or the Lorenz space and it is been shown $\mathbb{R}_{1}^{3}$.

Theorem 1. Let $X \in \mathbb{R}_{1}^{3}$ be any one vector.

(1) If $<\vec{X}, \vec{X}>_{L}>0$ or $\vec{X}=\overrightarrow{0}, \vec{X}$ is spacelike,

(2) If $<\vec{X}, \vec{X}>_{L}<0, \vec{X}$ is timelike,

(3) If $<\vec{X}, \vec{X}>_{L}<0, \vec{X}$ is lightlike (isotropic, null).

Definition 3. Minkowski space is a four-dimensional space possessing a Minkowski metric a metric tensor having the form

$$
d \tau^{2}=-\left(d x^{0}\right)^{2}+\left(d x^{1}\right)^{2}+\left(d x^{2}\right)^{2}+\left(d x^{3}\right)^{2}
$$

In equation (2) above, the metric signature $(1,3)$ is assumed; under this assumption, Minkowski space is typically written $\mathbb{R}^{(1,3)}$. One may also express equation (2) with respect to the metric signature $(3,1)$ by reversing the order of the positive and negative squared terms therein, in which case Minkowski space is denoted $\mathbb{R}^{(1,3)}$.

Definition 4. Suppose that $\xi$ is a vector field: that is, a vector-valued function with Cartesian coordinates $\left(\xi_{1}, \ldots, \xi_{n}\right)$; and $\mathbf{x}(t)$ a parametric curve with Cartesian coordinates $\left(x_{1}(t), \ldots, x_{n}(t)\right)$. Then $\mathbf{x}(t)$ is an integral curve of $\xi$ if it is a solution of the following autonomous system of ordinary differential equations: $\frac{d x_{1}}{d t}=\xi_{1}\left(x_{1}, \ldots, x_{n}\right), \ldots, \frac{d x_{n}}{d t}=\xi_{n}\left(x_{1}, \ldots, x_{n}\right)$. Such a system may be written as a single vector equation

$$
\xi(\mathbf{x}(t))=\mathbf{x}^{\prime}(t)=\frac{\partial}{\partial t}(\mathbf{x}(t)) .
$$




\section{$2.1 \mathrm{~J}$-Holomorphic curve}

Definition 5. Let be $V$ a vector space over $\mathbb{R}$. Let $M$ be a differentiable manifold of dimension $2 n$, and suppose $J$ is a differentiable vector bundle isomorphism $J_{x}: T_{x} M \rightarrow T_{x} M$.

(1) A (almost) complex structure on $M$ for $J^{2} d=-I d$.

(2) A (almost) paracomplex structure on $M$ for $J^{2} d=I d$.

(3) A tangent (exact) structure on $M$ for $J^{2} d=0$.

Where $J^{2}=J \circ J$, and $I$ is the identity (unit) operator on $V$ by the map $J: V \rightarrow V$ and $V=\mathbb{R}^{n} \oplus \mathbb{R}^{n}$.

Definition 6. A tangent structure $J$ on $M$ assigns to each $p \in M$ a linear map $J_{p}: T_{p} M \rightarrow T_{p} M$ that is smooth in $p$ and satisfies $J_{p}^{2} d=0$ for all $p$. The pair $(M, J)$ is called a tangent manifold.

Theorem 2. Any complex manifold $M$ is also an almost complex manifold.

Lemma 1. Let $M$ be a smooth manifold. If $M$ admits a complex structure A, then $M$ admits an almost complex structure J. Let $\operatorname{dim}_{\mathbb{C}} M=m$ and $(z, U)$ be any holomorphic chart inducing a coordinate frame $\partial x_{1}, \partial y_{1}, \ldots, \partial x_{m}, \partial y_{m}$. Then $J$ is given locally as

$$
J_{p}\left(\partial x_{i} p\right)=\partial y_{i} p, \quad J_{p}\left(\partial y_{i} p\right)=-\partial x_{i} p
$$

where $1 \leq i \leq m$ and $p \in U[16]$.

$J$-holomorphic curve is a smooth map from a Riemann surface into an almost complex manifold that satisfies the CauchyRiemann equation.

Definition 7. Let $(M, \omega)$ be a symplectic manifold of dimension $2 n$, and let $J \in J(M, \omega)$ be an $\omega$-compatible almost complex structure. Let $g_{J}(\cdot, \cdot) \equiv \omega(\cdot, J \cdot)$ be the corresponding Hermitian metric on $M$.

Definition 8. Let $\left(\sum, j\right)$ be a Riemann surface with complex structurej. A smooth map $u: \sum \rightarrow M$ is called a $(J, j)$ holomorphic map (or simply a J-holomorphic map) if $d u \circ j=J \circ d u$, or equivalently,

$$
\bar{\partial}_{J}(u)=\frac{1}{2}(d u+J \circ d u \circ j)=0
$$

The equation $\bar{\partial}_{J}(u)=0$ is a first order, non-linear equation of Cauchy-Riemann type.

\section{Twistor theory}

Twisted geometries are discrete geometries that plays a role in loop quantum gravity, where they appear in the semiclassical limit of spin networks and it maps the geometric objects of conventional $3+1$ space-time (Minkowski space) into geometric objects in a 4-dimensional space endowed with a Hermitian form of signature $(2,2)$. This space is called twistor space, and its complex valued coordinates are called twistors.

Definition 9. (Twistor space) If $(M, g)$ is an oriented Riemannian $2 n$-manifold then: $\mathbb{Z}$ is the bundle of g-orthogonal positive complex structures on the tangent spaces of $M$ and $\tau: \mathbb{Z} \rightarrow M$ is the bundle projection. The fibre $F=\tau^{-1}(x)$ is the space of $J \in \operatorname{End}\left(T_{x} M\right)$ such that $(a) J^{2}=-i d,(b) g(J v, J w)=g(v, w)$ for all $v, w \in T_{x} M$.

Theorem 3. (Eells-Salamon 1985, Twistor correspondence) Let $M$ be a Riemannian 2n-manifold. There is an almost complex $n(n+1)$-manifold $(\mathbb{Z}, J)$, the twistor space of $M$, and a map $\tau: \mathbb{Z} \rightarrow M$ such that (a) J-holomorphic curves in $\mathbb{Z}$ project (via $\tau$ ) to minimal surfaces in $M,(b)$ moreover any minimal surface arises this way. 


\subsection{Generalized Kähler structures}

Definition 10. Let $(M, J, g)$ be a $2 n$-dimensional almost complex manifold and $g$ is a metric, i.e. $J$ is an almost complex structure such that $J^{2} X=-X, g(J X, J Y)=-g(X, Y)$, for all vector fields $X, Y$ on $M$ and $g$ is a metric.

Definition 11. Let $M$ be a complex manifold with complex structure $J$ and compatible Riemannian metric $g=<., .>$ as in $\langle J X, J Y\rangle=\langle X, Y>$. The alternating 2-form $\omega(X, Y):=g(J X, Y)$ is called the associated Kähler form. We can retrieve $g$ from $\omega, g(X, Y)=\omega(X, J Y)$. We can say that $g$ is a Kähler metric and that $M$ is a Kähler manifold if $\omega$ is closed and $(M, g)$ is displayed in the form.

Definition 12. Let $M$ be a complex manifold. A Riemannian metric on $M$ is called Hermitian if it is compatible with the complex structure $J$ of $M,<J X, J Y>=<X, Y>$. Then the associated differential two-form $\omega$ defined by $\omega(X, Y)=<$ $J X, Y>$ is called the Kähler form. It turns out that $\omega$ is closed if and only if $J$ is parallel. Then M is called a Kähler manifold and the metric on M a Kähler metric. Kähler manifolds are modelled on complex Euclidean space [17].

Definition 13. Let $V$ be a $2 n$-dimensional real vector space and let $\left\{\frac{\partial}{\partial x_{i}}+\frac{\partial}{\partial y_{i}}\right\}, i=1, \ldots, 4 n$, be an orthonormal basis of the space $V \otimes V^{*}$ endowed with the neutral metric (6). Then $\frac{\partial}{\partial x_{1}}, \ldots, \frac{\partial}{\partial x_{2 n}}$ is a basis of $V$ and $\frac{\partial}{\partial y_{1}}, \ldots, \frac{\partial}{\partial y_{2 n}}$ is a basis of $V^{*}$.

Definition 14. Let $V \otimes V^{*}$ be a $n$-dimensional real vector space and $g$ a metric of signature $(p, q)$ on it, $p+q=n$. We shall say that an orthogonal basis $\left\{\frac{\partial}{\partial x_{1}}, \ldots, \frac{\partial}{\partial x_{n}}\right\}$ of $V \otimes V^{*}$ is orthonormal if $\left\|\frac{\partial}{\partial x_{1}}\right\|^{2}=\cdots=\left\|\frac{\partial}{\partial x_{p+q}}\right\|^{2}=-1$. If $n=2 m$ is an even number and $p=q=m$, the metric $g$ is usually called neutral. Recall that a complex structure $J$ on $V \otimes V^{*}$ is called compatible with the metric $g$, if the endomorphism $J$ is $g$-skew-symmetric. Suppose that $p=2 k$ and $q=2 l$, and let $J$ be a compatible complex structure on $V \otimes V^{*}$. Then it is easy to see by induction that there is an orthonormal basis $\left\{\frac{\partial}{\partial x_{1}}, \ldots, \frac{\partial}{\partial x_{n}}\right\}$ of $V \otimes V^{*}$ such that $J \frac{\partial}{\partial x_{2 i-1}}=\frac{\partial}{\partial x_{2 i}}, i=1, \ldots, k+l$.

Definition 15. Let $V$ be a $2 n$-dimensional real vector space and $V^{*}$ its dual space. Then the vector space $V \oplus V^{*}$ admits $\boldsymbol{a}$ natural neutral metric defined by

$$
\langle X+\xi, Y+\eta\rangle=\frac{1}{2}(\xi(Y)+\eta(X)), X, Y \in V \text { and } \xi, \eta \in V^{*}
$$

Let $V$ be a 2-dimensional real vector space and let $\left\{Q_{i}=\frac{\partial}{\partial x_{i}}+\frac{\partial}{\partial y_{i}}\right\}, 1 \leq i \leq 4$, be an orthonormal basis of the space $V \oplus V^{*}$ endowed with its natural neutral metric (6). Then

$$
\frac{\partial}{\partial x_{3}}=a_{11} \frac{\partial}{\partial x_{1}}+a_{12} \frac{\partial}{\partial x_{2}}, \frac{\partial}{\partial x_{4}}=a_{21} \frac{\partial}{\partial x_{1}}+a_{22} \frac{\partial}{\partial x_{2}}
$$

where $A=\left[a_{i j}\right]$ is an orthogonal matrix. If $\operatorname{det} A=1$, the basis $\left\{Q_{i}\right\}$ yields the canonical orientation of $V \oplus V^{*}$ and if $\operatorname{det} A=-1$ it yields the opposite one (proof see [7]).

Theorem 4. Let $V$ be a 2-dimensional real vector space. Take a basis $\left\{\frac{\partial}{\partial x_{1}}, \frac{\partial}{\partial x_{2}}\right\}$ of $V$ and let $\left\{\frac{\partial}{\partial y_{1}}\right.$, $\left.\frac{\partial}{\partial y_{2}}\right\}$ of $V^{*}$ be its dual basis. Then $\left\{Q_{1}=\frac{\partial}{\partial x_{1}}+\frac{\partial}{\partial y_{1}}, Q_{2}=\frac{\partial}{\partial x_{2}}+\frac{\partial}{\partial y_{2}}, Q_{3}=\frac{\partial}{\partial x_{3}}-\frac{\partial}{\partial y_{3}}, Q_{4}=\frac{\partial}{\partial x_{4}}-\frac{\partial}{\partial y_{4}}\right\}$ is an orthonormal basis of $V \otimes V^{*}$ with respect to the natural neutral metric (2) and is positively oriented with respect to the canonical orientation of $V \otimes V^{*}$. Set $\varepsilon_{k}=\left\|Q_{k}\right\|^{2}, k=1, \ldots, 4$, and define skew-symmetric endomorphisms of $V \otimes V^{*}$ setting $S_{i j} Q_{k}=\varepsilon_{k}\left(\delta_{i k} Q_{j}-\delta_{i k} Q\right)$, $1 \leq i, j, k \leq 4$. Then the endomorphisms

$$
\begin{aligned}
& I_{1}=S_{12}-S_{34}, J_{1}=S_{12}+S_{34}, \\
& I_{2}=S_{13}-S_{24}, J_{2}=S_{13}+S_{24}, \\
& I_{3}=S_{14}+S_{23}, J_{3}=S_{14}-S_{23},
\end{aligned}
$$


constitute a basis of the space of skew-symmetric endomorphisms of $V \otimes V^{*}$ subject to the following relations:

$$
\begin{array}{ll}
I_{1}^{2}=I_{2}^{2}=I d, I_{3}^{2}=0, & \\
J_{1}^{2}=J_{2}^{2}=I d, J_{3}^{2}=0 & \\
I_{r} I_{s}=-I_{s} I_{r}, J_{r} J_{s}=-J_{s} J_{r}, & 1 \leqslant r \neq s \leqslant 3 \\
I_{r} J_{s}=J_{s} I_{r}, & 1 \leqslant r, s \leqslant 3 .
\end{array}
$$

Let $\Psi$ be a complex structure on $V \otimes V^{*}$ compatible with the metric and let us set $\Psi=\sum_{r=1}^{3}\left(x_{r} I_{r}+y_{r} J_{r}\right)$. Then we have $\Psi^{2}=I d$ if and only if either $I=\sum_{r} x_{r} I_{r}$ with $x_{1}^{2}+x_{2}^{2}=1$ and $y_{1}=y_{2}=y_{3}=0, J=\sum_{s} y_{s} J_{s}$ with $y_{1}^{2}+y_{2}^{2}=1$ and $x_{1}=x_{2}=x_{3}=0$. It follows that

$$
\begin{gathered}
I \frac{\partial}{\partial x_{1}}=x_{1} \frac{\partial}{\partial x_{1}}+\left(x_{2}+1\right) \frac{\partial}{\partial x_{2}}, J \frac{\partial}{\partial x_{1}}=y_{1} \frac{\partial}{\partial x_{1}}+\left(y_{2}-1\right) \frac{\partial}{\partial y_{2}}, \\
I \frac{\partial}{\partial x_{2}}=\left(x_{2}-1\right) \frac{\partial}{\partial x_{1}}-x_{1} \frac{\partial}{\partial x_{2}}, J \frac{\partial}{\partial x_{2}}=y_{1} \frac{\partial}{\partial x_{2}}+\left(y_{2}-1\right) \frac{\partial}{\partial y_{1}}, \\
I \frac{\partial}{\partial y_{1}}=-x_{1} \frac{\partial}{\partial y_{1}}-\left(x_{2}-1\right) \frac{\partial}{\partial y_{2}}, J \frac{\partial}{\partial y_{1}}=\left(y_{2}+1\right) \frac{\partial}{\partial x_{2}}-y_{1} \frac{\partial}{\partial y_{1}} \\
I \frac{\partial}{\partial y_{2}}=-\left(x_{2}+1\right) \frac{\partial}{\partial y_{1}}+x_{1} \frac{\partial}{\partial y_{2}}, J \frac{\partial}{\partial y_{2}}=\left(y_{2}+1\right) \frac{\partial}{\partial x_{1}}-y_{1} \frac{\partial}{\partial y_{2}} .
\end{gathered}
$$

This shows that the restriction of $I$ to $V$ is a complex structure on $V$ inducing the generalized complex structure $I$. In contrast, the generalized complex structure $J$ is not induced by a complex structure or a symplectic form on $V$.

Proof. Let us set $\Psi=\sum_{r=1}^{3}\left(x_{r} I_{r}+y_{r} J_{r}\right)$. Let's we have account $\Psi^{2}$ using Definition 5 :

$$
\begin{aligned}
& \Psi^{2}=\sum_{r=1}^{3}\left(x_{r} I_{r}+y_{r} J_{r}\right)^{2} \\
& =x_{1}^{2} I_{1}^{2}+x_{2}^{2} I_{2}^{2}+x_{3}^{2} I_{3}^{2}+y_{1}^{2} J_{1}^{2}+J_{2}^{2} y_{2}^{2}+J_{3}^{2} y_{3}^{2}+2 \sum_{r, s=1}^{3}\left(x_{r} y_{s} I_{r} J_{s}\right) . \\
& =x_{1}^{2}+x_{2}^{2}+y_{1}^{2}+y_{2}^{2}+2 \sum_{r, s=1}^{3}\left(x_{r} y_{s} I_{r} J_{s}\right) .
\end{aligned}
$$

We see that $x_{1}^{2}+x_{2}^{2}+y_{1}^{2}+y_{2}^{2}=1, x_{r} y_{s}=0, r, s=1,2,3$. Therefore $\Psi^{2}=I d$ if and only if either $x_{1}^{2}+x_{2}^{2}=1$ for $y_{1}=y_{2}=$ $y_{3}=0$ and $y_{1}^{2}+y_{2}^{2}=1$ for $x_{1}=x_{2}=x_{3}=0$ [7].

Theorem 5. If $\alpha$ and $\beta$ are 1-forms, then $\alpha \wedge \beta$ is a 2-forms.

Definition 16. In three dimensions, the vector from the origin to the point with Cartesian coordinates $(x, y, z)$ can be written as [18]:

$$
r=x \mathbf{i}+y \mathbf{j}+z \mathbf{k}=x\left(\frac{\partial}{\partial x}\right)+y\left(\frac{\partial}{\partial y}\right)+z\left(\frac{\partial}{\partial z}\right) .
$$

Proposition 1. Let $\vec{X}, \mathbf{Y} \in \mathbb{R}_{1}^{3}$ be any two vector that are. $\vec{X}=(x, y+1,0), \mathbf{Y}=(y-1,-x, 0)$. Let this curve expressed as vectors:

$$
r_{1}=x\left(\frac{\partial}{\partial x}\right)+(y+1)\left(\frac{\partial}{\partial y}\right), r_{2}=(y-1)\left(\frac{\partial}{\partial x}\right)-x\left(\frac{\partial}{\partial y}\right) .
$$

Example 1. Let us consider the structure (10) using the holomorphic property at (13). We can define the following holomorphic structures with:

$$
\begin{aligned}
& \text { (1) } J \frac{\partial}{\partial x}=x \frac{\partial}{\partial x}+(y+1) \frac{\partial}{\partial y}, \\
& \text { (2) } J \frac{\partial}{\partial y}=(y-1) \frac{\partial}{\partial x}-x \frac{\partial}{\partial y} .
\end{aligned}
$$

Now, we denote the structure (14) of the holomorphic property:

$$
\begin{aligned}
& \text { (1) } J^{2} \frac{\partial}{\partial x}=x\left(x \frac{\partial}{\partial x}+(y+1) \frac{\partial}{\partial y}\right)+(y+1)\left((y-1) \frac{\partial}{\partial x}-x \frac{\partial}{\partial y}\right)=\left(x^{2}+y^{2}-1\right) \frac{\partial}{\partial x} . \\
& \text { (2) } J^{2} \frac{\partial}{\partial y}=(y-1)\left(x \frac{\partial}{\partial x}+(y+1) \frac{\partial}{\partial y}\right)-x\left((y-1) \frac{\partial}{\partial x}-x \frac{\partial}{\partial y}\right)=\left(x^{2}+y^{2}-1\right) \frac{\partial}{\partial y} .
\end{aligned}
$$


As shown above, the structures (15) are tangent (Defintion5) and $x^{2}+y^{2}=1$ for $J^{2}=0$. This is a cone and the graph is as follows:

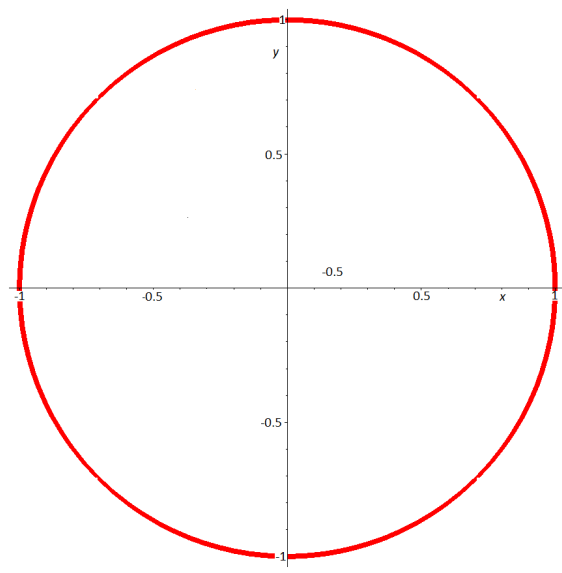

\section{4 (Euler)-Lagrange dynamics equations}

Lemma 2. The closed 2-form on a vector field and 1-form reduction function on the phase space defined of a mechanical system is equal to the differential of the energy function 1-form of the Lagrangian and the Hamiltonian mechanical systems $[19,20]$.

Definition 17. Let $M$ be an n-dimensional manifold and TM its tangent bundle with canonical projection $\tau_{M}: T M \rightarrow M$. $T M$ is called the phase space of velocities of the base manifold $M$. Let $L: T M \rightarrow R$ be a differentiable function on TM called the Lagrangian function. Here, $L=T-V$ such that $T$ is the kinetic energy and $V$ is the potential energy of a mechanical system. In the problem of a mass on the end of a spring, $T=m \dot{x}^{2} / 2$ and $V=k x^{2} / 2$.

Definition 18. We consider the closed 2-form and base space $(J)$ on TM given by $\Phi_{L}=-d\left(\mathbf{d}_{J} L\right)=-d(J(\mathbf{d}))$. Consider the equation

$$
i_{\xi} \Phi_{L}=d E_{L}
$$

Where $i_{\xi}$ is reduction function and $i_{\xi} \Phi_{L}=\Phi_{L}(\xi)$ is defined in the form. Then $\xi$ is a vector field, we shall see that (16) under a certain condition on $\xi$ is the intrinsical expression of the Euler-Lagrange equations of motion. This equation (16) is named as Lagrange dynamical equation [21,22].

Definition 19. We shall see that for motion in a potential, $E_{L}=V L-L$ is an energy function and $V=J \xi$ a Liouville vector field. Here $d E_{L}$ denotes the differential of $E$. The triple $\left(T M, \Phi_{L}, \xi\right)$ is known as Lagrangian system on the tangent bundle TM. If it is continued the operations on (16) for any coordinate system then infinite dimension Lagrange's equation is obtained the form below. The equations of motion in Lagrangian mechanics are the Lagrange equations of the second kind, also known as the Euler-Lagrange equations;

$$
\frac{\partial}{\partial t}\left(\frac{\partial L}{\partial \dot{x}}\right)=\frac{\partial L}{\partial x}
$$




\section{Euler-Lagrangian mechanical equations}

Let's get, using (16), Euler-Lagrange equations on twistorial generalized Kähler manifolds and its shown that by $(T M, g, J)$.

Proposition 2. Let $\xi$ be the vector field determined by

$$
\xi=X \frac{\partial}{\partial x}+Y \frac{\partial}{\partial y}, X=\dot{x}, Y=\dot{y}
$$

on $(T M, g, J)$.

Then the vector field defined by

$$
V=J(\xi)=J\left(X \frac{\partial}{\partial x}+Y \frac{\partial}{\partial y}\right)
$$

is thought to be Liouville vector field on twistorial generalized Kähler manifolds $(T M, g, J) . \Phi_{L}=-d\left(\mathbf{d}_{J} L\right), d=\frac{\partial}{\partial x} d x+$ $\frac{\partial}{\partial y} d y$ is the closed 2-form given by (16) such that

$$
\begin{aligned}
& \mathbf{d}=\frac{\partial}{\partial x} d x+\frac{\partial}{\partial y} d y, \quad \mathbf{d}_{J}: F(M) \rightarrow \wedge^{1} M, \\
& \mathbf{d}_{J}=J\left(\frac{\partial}{\partial x} d x+\frac{\partial}{\partial y} d y\right),
\end{aligned}
$$

and $\mathbf{d}_{J}=i_{J} \mathbf{d}-\mathbf{d} i_{J}$. Also, the vertical differentiation $\mathbf{d}_{J}$ is given by $\mathrm{d}$ is the usual exterior derivation. Then there is the following result. Here, we can be account Euler-Lagrange equations for classical and analytical mechanics on twistorial generalized Kähler manifolds $(T M, g, J)$. We get the equations given by

$$
\mathbf{d}_{J} L=\left[x \frac{\partial L}{\partial x}+(y+1) \frac{\partial L}{\partial y}\right] d x+\left[(y-1) \frac{\partial L}{\partial x}-x \frac{\partial L}{\partial y}\right] d y
$$

Let we account $\Phi_{L}$ :

$$
\begin{aligned}
\Phi_{L}= & -d\left(\mathbf{d}_{J} L\right)=-d\left(\left[x \frac{\partial L}{\partial x}+(y+1) \frac{\partial L}{\partial y}\right] d x+\left[(y-1) \frac{\partial L}{\partial x}-x \frac{\partial L}{\partial y}\right] d y\right) \\
= & {\left[\frac{\partial L}{\partial x}+x \frac{\partial^{2} L}{\partial x \partial x}+(y+1) \frac{\partial^{2} L}{\partial x \partial y}\right] d x \wedge d x+\left[(y-1) \frac{\partial^{2} L}{\partial x \partial x}-\frac{\partial L}{\partial y}-x \frac{\partial^{2} L}{\partial x \partial y}\right] d y \wedge d x } \\
& +\left[x \frac{\partial^{2} L}{\partial y \partial x}+\frac{\partial L}{\partial y}+(y+1) \frac{\partial^{2} L}{\partial y \partial y}\right] d x \wedge d y+\left[\frac{\partial L}{\partial x}+(y-1) \frac{\partial^{2} L}{\partial y \partial x}-x \frac{\partial^{2} L}{\partial y \partial y}\right] d y \wedge d y .
\end{aligned}
$$

Then we find using $(f \wedge g)(v)=f(v) g-g(v) f,\left(d x_{i} \wedge d x_{j}\right)\left(\frac{\partial}{\partial x_{k}}\right)=d x_{i} \frac{\partial}{\partial x_{k}} d x_{j}-d x_{j} \frac{\partial}{\partial x_{k}} d x_{i}=\frac{\partial x_{i}}{\partial x_{k}} d x_{j}-\frac{\partial x_{j}}{\partial x_{k}} d x_{i}, \frac{\partial x_{i}}{\partial x_{k}}=0$, $\frac{\partial x_{i}}{\partial x_{i}}=1$

$$
\begin{aligned}
& \Phi_{L}(\xi)=X\left[\frac{\partial L}{\partial x}+x \frac{\partial^{2} L}{\partial x \partial x}+(y+1) \frac{\partial^{2} L}{\partial x \partial y}\right]\left[d x \frac{\partial}{\partial x} d x-d x \frac{\partial}{\partial x} d x\right] \\
& +X\left[(y-1) \frac{\partial^{2} L}{\partial x \partial x}-\frac{\partial L}{\partial y}-x \frac{\partial^{2} L}{\partial x \partial y}\right]\left[d y \frac{\partial}{\partial x} d x-d x \frac{\partial}{\partial x} d y\right] \\
& +X\left[x \frac{\partial^{2} L}{\partial y \partial x}+\frac{\partial L}{\partial y}+(y+1) \frac{\partial^{2} L}{\partial y \partial y}\right]\left[d x \frac{\partial}{\partial x} d y-d y \frac{\partial}{\partial x} d x\right] \\
& +X\left[\frac{\partial L}{\partial x}+(y-1) \frac{\partial^{2} L}{\partial y \partial x}-x \frac{\partial^{2} L}{\partial y \partial y}\right]\left[d y \frac{\partial}{\partial x} d y-d y \frac{\partial}{\partial x} d y\right. \\
& +Y\left[\frac{\partial L}{\partial x}+x \frac{\partial^{2} L}{\partial x \partial x}+(y+1) \frac{\partial^{2} L}{\partial x \partial y}\right]\left[d x \frac{\partial}{\partial y} d x-d x \frac{\partial}{\partial y} d x\right] \\
& +Y\left[(y-1) \frac{\partial^{2} L}{\partial x \partial x}-\frac{\partial L}{\partial y}-x \frac{\partial^{2} L}{\partial x \partial y}\right]\left[d y \frac{\partial}{\partial y} d x-d x \frac{\partial}{\partial y} d y\right] \\
& +Y\left[x \frac{\partial^{2} L}{\partial y \partial x}+\frac{\partial L}{\partial y}+(y+1) \frac{\partial^{2} L}{\partial y \partial y}\right]\left[d x \frac{\partial}{\partial y} d y-d y \frac{\partial}{\partial y} d x\right] \\
& +Y\left[\frac{\partial L}{\partial x}+(y-1) \frac{\partial^{2} L}{\partial y \partial x}-x \frac{\partial^{2} L}{\partial y \partial y}\right]\left[d y \frac{\partial}{\partial y} d y-d y \frac{\partial}{\partial y} d y\right] \text {. }
\end{aligned}
$$


from here

$$
\begin{aligned}
& \Phi_{L}(\xi)=X\left[\frac{\partial L}{\partial x}+x \frac{\partial^{2} L}{\partial x \partial x}+(y+1) \frac{\partial^{2} L}{\partial x \partial y}\right] d x-X\left[\frac{\partial L}{\partial x}+x \frac{\partial^{2} L}{\partial x \partial x}+(y+1) \frac{\partial^{2} L}{\partial x \partial y}\right] d x \\
& \quad-X\left[(y-1) \frac{\partial^{2} L}{\partial x \partial x}-\frac{\partial L}{\partial y}-x \frac{\partial^{2} L}{\partial x \partial y}\right] d y+X\left[x \frac{\partial^{2} L}{\partial y \partial x}+\frac{\partial L}{\partial y}+(y+1) \frac{\partial^{2} L}{\partial y \partial y}\right] d y \\
& +Y\left[(y-1) \frac{\partial^{2} L}{\partial x \partial x}-\frac{\partial L}{\partial y}-x \frac{\partial^{2} L}{\partial x \partial y}\right] d x-Y\left[x \frac{\partial^{2} L}{\partial y \partial x}+\frac{\partial L}{\partial y}+(y+1) \frac{\partial^{2} L}{\partial y \partial y}\right] d x \\
& +Y\left[\frac{\partial L}{\partial x}+(y-1) \frac{\partial^{2} L}{\partial y \partial x}-x \frac{\partial^{2} L}{\partial y \partial y}\right] d y-Y\left[\frac{\partial L}{\partial x}+(y-1) \frac{\partial^{2} L}{\partial y \partial x}-x \frac{\partial^{2} L}{\partial y \partial y}\right] d y .
\end{aligned}
$$

Also, the energy function of system is

$$
E_{L}=V L-L=J\left(X \frac{\partial L}{\partial x}+Y \frac{\partial L}{\partial y}\right)-L=X\left[x \frac{\partial L}{\partial x}+(y+1) \frac{\partial L}{\partial y}\right]+Y\left[(y-1) \frac{\partial L}{\partial x}-x \frac{\partial L}{\partial y}\right]-L
$$

and the differential of $E_{L}$ is

$$
\begin{aligned}
& d E_{L}=d\left(X\left[x \frac{\partial L}{\partial x}+(y+1) \frac{\partial L}{\partial y}\right]+Y\left[(y-1) \frac{\partial L}{\partial x}-x \frac{\partial}{\partial y} L\right]-L\right) \\
& =X\left(\frac{\partial L}{\partial x} d x+x \frac{\partial^{2} L}{\partial x \partial x} d x+(y+1) \frac{\partial^{2} L}{\partial x \partial y} d x\right)+Y\left((y-1) \frac{\partial^{2} L}{\partial x \partial x} d x-\frac{\partial L}{\partial y} d x-x \frac{\partial^{2} L}{\partial x \partial y} d x\right)-\frac{\partial L}{\partial x} d x \\
& +X\left(x \frac{\partial^{2} L}{\partial y \partial x} d y+\frac{\partial L}{\partial y} d y+(y+1) \frac{\partial^{2} L}{\partial y \partial y} d y\right)+Y\left(\frac{\partial L}{\partial x} d y+(y-1) \frac{\partial^{2} L}{\partial y \partial x} d y-x \frac{\partial^{2} L}{\partial y \partial y} d y\right)-\frac{\partial L}{\partial y} d y .
\end{aligned}
$$

Using (4), we find the following equations:

$$
\begin{aligned}
& -X\left[\frac{\partial L}{\partial x}+x \frac{\partial^{2} L}{\partial x \partial x}+(y+1) \frac{\partial^{2} L}{\partial x \partial y}\right] d x-Y\left[x \frac{\partial^{2} L}{\partial y \partial x}+\frac{\partial L}{\partial y}+(y+1) \frac{\partial^{2} L}{\partial y \partial y}\right] d x+\frac{\partial L}{\partial x} d x=0, \\
& -X\left[(y-1) \frac{\partial^{2} L}{\partial x \partial x}-\frac{\partial L}{\partial y}-x \frac{\partial^{2} L}{\partial x \partial y}\right] d y-Y\left[\frac{\partial L}{\partial x}+(y-1) \frac{\partial^{2} L}{\partial y \partial x}-x \frac{\partial^{2} L}{\partial y \partial y}\right] d y+\frac{\partial L}{\partial y} d y=0,
\end{aligned}
$$

or

$$
\begin{aligned}
& -x\left[X \frac{\partial}{\partial x}+Y \frac{\partial}{\partial y}\right] \frac{\partial L}{\partial x}-(y+1)\left[X \frac{\partial}{\partial x}+Y \frac{\partial}{\partial y}\right] \frac{\partial L}{\partial y}+\frac{\partial L}{\partial x}=0 \\
& -(y-1)\left[X \frac{\partial}{\partial x}+Y \frac{\partial}{\partial y}\right] \frac{\partial L}{\partial x}+x\left[X \frac{\partial}{\partial x}+Y \frac{\partial}{\partial y}\right] \frac{\partial L}{\partial y}+\frac{\partial L}{\partial y}=0
\end{aligned}
$$

The above equation (28), using Definition 8, arranged on the result below:

$$
\begin{aligned}
& \text { dif } 1 .-x \frac{\partial}{\partial t}\left(\frac{\partial L}{\partial x}\right)-(y+1) \frac{\partial}{\partial t}\left(\frac{\partial L}{\partial y}\right)+\frac{\partial L}{\partial x}=0 \\
& \text { dif2. }-(y-1) \frac{\partial}{\partial t}\left(\frac{\partial L}{\partial x}\right)+x \frac{\partial}{\partial t}\left(\frac{\partial L}{\partial y}\right)+\frac{\partial L}{\partial y}=0
\end{aligned}
$$

such that the differential equations (29) are named Euler-Lagrange mechanical equations on twistorial generalized Kähler manifolds such that this is shown in the form of $(T M, g, J)$. Additionally, therefore the triple $\left(T M, \Phi_{L}, \xi\right)$ is called a Euler-Lagrangian mechanical system on $(T M, g, J)$.

\section{Equations solving with computer}

Set out in this study (29) equations are partial differential equations on twistorial generalized Kähler manifolds such that its solved with Maple computation program.

Example 2. Implicit solution (29) is $L(x, y, t)=F_{1}(t)$. 


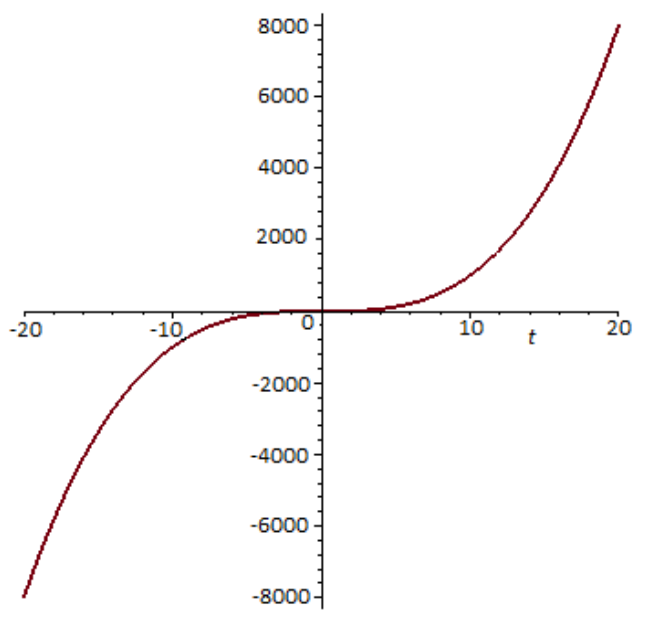

Fig. 1: Special values of $F_{1}(t)=t^{3}$.

\section{Discussion}

It is well-known that a classical field theory explain the study of how one or more physical fields interact with matter which is used quantum and classical mechanics of physics branches. How the movement of objects in electrical, magnetically and gravitational fields force is very important. Because, the classical theory of electromagnetism deals with electric and magnetic fields and their interaction with each other and with charges and currents. An electromagnetic field is a physical field produced by electrically charged objects and their locations over time.

It is well-known that the motivation and one of the initial aims of twistor theory is to provide an adequate formalism for the union of quantum theory and general relativity. Twistor theory can also be used to solve non-linear differential equations which are related to the self-duality equations that describe instantaneous in $\mathbb{R}^{4}$. In this study, the Euler-Lagrange mechanical equations (29) derived on twistorial generalized Kähler manifolds may be suggested to deal with problems in electrical, magnetically and gravitational fields for the path of movement (Fig 1.) of defined space moving objects $[23,24]$.

\section{References}

[1] R. Penrose, Twistor algebra. J. Math. Phys., 8, (1967), 345-366.

[2] R. Penrose, Twistor Theory, Its Aims and Achievements, Clarendon Press, Oxford, (1975), 268-407.

[3] L. Freidela and S. Spezialeb, From Twistors to Twisted Geometries, http://arxiv.org/abs/1006.0199v1, (2010).

[4] S. Speziale, Loop Quantum Gravity and Twisted Geometries, Zakopane, (2011).

[5] S. Santa-Cruz, Twistor Geometry for Hyperkahler Metrics on Complex Adjoint Orbits, Annals of Global Analysis and Geometry 15: 361-377, 1997.

[6] R. Albuquerque, Twistorial Constructions of Special Riemannian Manifolds, AIP Conference Proceedings, Vol. 1023 Issue 1, (2008).

[7] J. Davidov and O. Mushkarov, Twistor Spaces of Generalized Complex Structures, JGP, 56, (2006), 1623-1636.

[8] E. Loubeau and R. Pantilie, Harmonic Morphisms Between Weyl Spaces and Twistorial Maps, Communications in Analysis and Geometry, Volume 14, Number 5, 847-881, 2006 
[9] R. Pantilie, On a Class of Twistorial Maps, Differential Geometry and its Applications, 26, (2008), 366-376.

[10] S. Ianuss S. Marchiafava, L. Ornea, R. Pantilie, Twistorial Maps Between Quaternionic Manifolds, arXiv:0801.4587v2, (2008).

[11] M. Dunajski, Twistor Theory and Differential Equations, arXiv:0902.0274v2, (2009).

[12] S. Marchiafava, Twistorial Maps Between (para) Quaternionic Projective Spaces, Bull. Math. Soc. Sci. Math. Roumanie Tome, 52(100), No. 3, (2009), 321-332.

[13] Z. Kasap, Weyl-Euler-Lagrange Equations of Motion on Flat Manifold, Advances in Mathematical Physics, (2015), 1-16.

[14] M. Tekkoyun, Mechanics Systems on Para-Kählerian Manifolds of Constant J-Sectional Curvature, http://arxiv.org/abs/0902.3569v1, (2009), 1-8.

[15] S. Cecotti, A. Neitzke, C. Vafa, Twistorial Topological Strings and a tt* Geometry for N=2 Theories in 4d, arXiv:1412.4793v1, (2014).

[16] N. Nowaczyk, J. Niediek and M. Firsching, Basics of Complex Manifolds, (2009).

[17] W. Ballmann, Lectures on Kähler Manifolds, ESI Lectures in Mathematics and Physics, ISBN 978-3-03719-025-8, (2006).

[18] D.J. Griffiths, Introduction to Electrodynamics. Prentice Hall. ISBN 0-13-805326-X, (1999).

[19] J. Klein, Escapes Variationnels et Mécanique, Ann. Inst. Fourier, Grenoble, 12 (1962).

[20] A. Ibord, The Geometry of Dynamics, Extracta Mathematicable, Vol.11, Num.1, (1996), 80-105.

[21] M. de Leon and P.R. Rodrigues, Methods of Differential Geometry in Analytical Mechanics, Elsevier Sc. Pub. Com. Inc., Amsterdam, (1989).

[22] R. Abraham, J.E. Marsden and T. Ratiu, Manifolds Tensor Analysis and Applications, Springer, (2001).

[23] B. Thidé, Electromagnetic Field Theory, (2012).

[24] R.G. Martín Electromagnetic Field Theory for Physicists and Engineers: Fundamentals and Applications, Granada, (2007). 